\title{
Cross Helicity Reversals in Magnetic Switchbacks
}

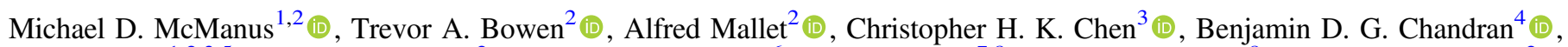

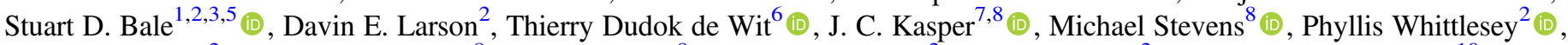

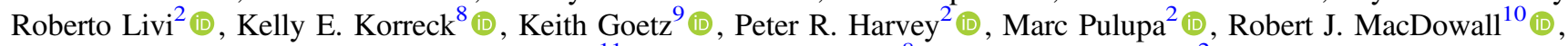 \\ David M. Malaspina ${ }^{11}$ (1) , Anthony W. Case ${ }^{8}$ (10) and J. W. Bonnell ${ }^{2}$ (1) \\ ${ }^{1}$ Physics Department, University of California, Berkeley, CA 94720-7300, USA; mdmcmanus@ berkeley.edu \\ 2 Space Sciences Laboratory, University of California, Berkeley, CA 94720-7450, USA \\ ${ }^{3}$ School of Physics and Astronomy, Queen Mary University of London, London E1 4NS, UK \\ ${ }^{4}$ Department of Physics and Astronomy, University of New Hampshire, Durham, NH 03824, USA \\ ${ }^{5}$ The Blackett Laboratory, Imperial College London, London, SW7 2AZ, UK \\ ${ }^{6}$ LPC2E, CNRS and University of Orléans, Orléans, France \\ ${ }^{7}$ Climate and Space Sciences and Engineering, University of Michigan, Ann Arbor, MI 48109, USA \\ ${ }^{8}$ Smithsonian Astrophysical Observatory, Cambridge, MA 02138, USA \\ ${ }^{9}$ School of Physics and Astronomy, University of Minnesota, Minneapolis, MN 55455, USA \\ ${ }^{10}$ Solar System Exploration Division, NASA/Goddard Space Flight Center, Greenbelt, MD 20771, USA \\ ${ }^{11}$ Laboratory for Atmospheric and Space Physics, University of Colorado, Boulder, CO 80303, USA \\ Received 2019 September 21; revised 2020 January 15; accepted 2020 January 19; published 2020 February 6
}

\begin{abstract}
We consider 2D joint distributions of normalized residual energy, $\sigma_{r}(s, t)$, and cross helicity, $\sigma_{c}(s, t)$, during one day of Parker Solar Probe's (PSP's) first encounter as a function of wavelet scale $s$. The broad features of the distributions are similar to previous observations made by Helios in slow solar wind, namely well-correlated and fairly Alfvénic wind, except for a population with negative cross helicity that is seen at shorter wavelet scales. We show that this population is due to the presence of magnetic switchbacks, or brief periods where the magnetic field polarity reverses. Such switchbacks have been observed before, both in Helios data and in Ulysses data in the polar solar wind. Their abundance and short timescales as seen by PSP in its first encounter is a new observation, and their precise origin is still unknown. By analyzing these MHD invariants as a function of the wavelet scale, we show that magnetohydrodynamic (MHD) waves do indeed follow the local mean magnetic field through switchbacks, with a net Elsässer flux propagating inward during the field reversal and that they, therefore, must be local kinks in the magnetic field and not due to small regions of opposite polarity on the surface of the Sun. Such observations are important to keep in mind as computing cross helicity without taking into account the effect of switchbacks may result in spurious underestimation of $\sigma_{c}$ as PSP gets closer to the Sun in later orbits.
\end{abstract}

Unified Astronomy Thesaurus concepts: Heliosphere (711); Interplanetary turbulence (830); Space plasmas (1544); Alfven waves (23)

\section{Introduction}

Parker Solar Probe (PSP; Fox et al. 2016) was launched in 2018 August with the aim of shedding light on the plasma and magnetic field environments of the inner heliosphere and the longstanding problem of coronal heating. It completed its first of a series of 24 encounters on 2018 November 11, during which at perihelion it was a distance of $35 R_{S}$ from the Sun.

One of the more notable observations reported from the first encounter has been the preponderance of so-called magnetic "switchbacks," which are large traversals of the mainly radial magnetic field, often temporarily reversing the sense of the field. Prior to $P S P$, magnetic switchbacks had been observed both in near-Sun (0.3 au) Helios data (Horbury et al. 2018) and over the solar poles by Ulysses (Balogh et al. 1999). Both studies involved fast solar wind streams. After reprocessing Helios data, Horbury et al. (2018) found that large velocity spikes are ubiquitous in near-Sun fast solar wind, occurring about $5 \%$ of the time and with magnitudes of the order of $0.5 v_{A}$ above the background solar wind speed. The velocity spikes they observed were almost always positive speed enhancements, were highly Alfvénic in all three components (thus by necessity accompanied by large magnetic field traversals), and showed no statistically meaningful difference in plasma parameters inside versus outside the spikes (making it unlikely that the observed field geometry was due to Helios crossing large coronal loops). Those authors speculated that they may be the Alfvénic fluctuations that travel ahead of jets generated by reconnection events in the corona (Karpen et al. 2017; Uritsky et al. 2017) and are thus signatures of transient events at the Suns surface that have survived to relatively large distances. The spikes or switchbacks seen by PSP in its first two encounters are qualitatively different than these in two ways; they are shorter in timescale (presumably due to being at smaller heliocentric distances and having better measurement cadences able to resolve sharper spikes), and they are the first direct observation of them in slow as opposed to fast solar wind, marking them to be a universal feature of the solar wind.

Earlier work by Balogh et al. (1999) reported magnetic field inversions at high heliographic latitudes that lasted of the order of several hours and used cross helicity as a sensor of the wave propagation direction to deduce that the inversions they saw were not intrinsically different magnetic sectors but rather due to fold-like structures in the magnetic field. In this work, we use wavelet representations of the dimensionless magnetohydrodynamic (MHD) transport ratios cross helicity, $\sigma_{c}$, and residual energy, $\sigma_{r}$, in a similar way to probe the geometry of the short timescale magnetic switchbacks seen by PSP in encounter 1 over a wide range of scales. We deduce that they 
too are due to localized folds in the magnetic field and not regions of different magnetic polarity.

Several other sensors can be used to elucidate local magnetic field topology. Electron strahl pitch angle distributions, as measured by the Solar Probe Analyzers (SPAN) instrument on PSP (Livi et al. 2019; Whittlesey et al. 2019b), are used by Whittlesey et al. (2019a) to follow the magnetic field through switchbacks. Neugebauer \& Goldstein (2013) showed that the relative proton core-beam drift becomes negative (that is, the beam appears to be moving more slowly than the core in the spacecraft frame) whenever the local field switches back on itself, and Yamauchi et al. (2004) used the alpha-proton differential velocity to show the same thing within the context of pressure balance structures. Our technique has the advantage of being somewhat less complex than these methods, requiring a less detailed analysis of the particle distribution functions (only the perturbed bulk velocity moments are needed). It is worth mentioning that the wavelet method of analyzing MHD transport ratios employed here is a very versatile one and can be used to identify other structures in the solar wind. Zhao et al. (2020) use a similar technique to identify and catalog smallscale flux ropes during and around PSP's first encounter.

This clear dependence of plasma properties on the local magnetic field is reflected in the plasma turbulence as well. Turbulent power is concentrated at near perpendicular angles $\theta_{B V}$ between the magnetic field and flow direction, and the magnetic field spectral index is a smoothly increasing function of $\theta_{B V}$ (Horbury et al. 2008; Podesta 2009; Chen et al. 2011). This dependence of the spectral index on $\theta_{B V}$ was only revealed when sufficient care was used to examine the mean field at small enough (i.e., localized) scales, via a wavelet method.

Throughout the solar wind, we see Alfvénic turbulence, and there are numerous models of how this turbulence behaves both at 1 au (Boldyrev 2005; Mallet \& Schekochihin 2016) and in the inner heliosphere (Velli et al. 1989; Perez \& Chandran 2013; Chandran \& Perez 2019). The relationship between $\sigma_{c}$ and $\sigma_{r}$, as useful invariants to characterize the state of the MHD turbulence, has been well studied (Bruno \& Carbone 2005; Bruno et al. 2007 and references therein). Fast wind at short heliocentric distances is very Alfvénic and equipartitioned $\left(\sigma_{c} \sim 1, \sigma_{r} \sim 0\right)$, but a second population with $\sigma_{c} \sim 0, \sigma_{r} \sim$ -1 appears as heliocentric distance increases, representing the presence of intermittent magnetic structures. The importance of negative residual energy and intermittency and how it causes the magnetic field spectrum to steepen was highlighted in Bowen et al. (2018) and Chen et al. (2013). Slow wind does not show such marked radial evolution, with broader $\left(\sigma_{c}\right.$ and $\left.\sigma_{r}\right)$ distributions in general.

In Section 2, we outline the data set and methods used. Section 3 contains results and discussion, and we briefly summarize the conclusions in Section 4.

\section{Data and Methods}

We use particle measurements of proton density $\rho$ and velocity $v$ made by PSP's onboard Faraday cup, Solar Probe Cup (SPC; Kasper et al. 2016) and magnetic field measurements made by the FIELDS fluxgate magnetometer (Bale et al. 2016). We consider a 1 day interval from encounter 1, 2018 November 5. The encounter 1 measurement cadence for SPC proton moments is approximately $0.87 \mathrm{~s}$, while the magnetometer measurement frequency was approximately $293 \mathrm{~Hz}$. The magnetometer data was downsampled to match SPC's
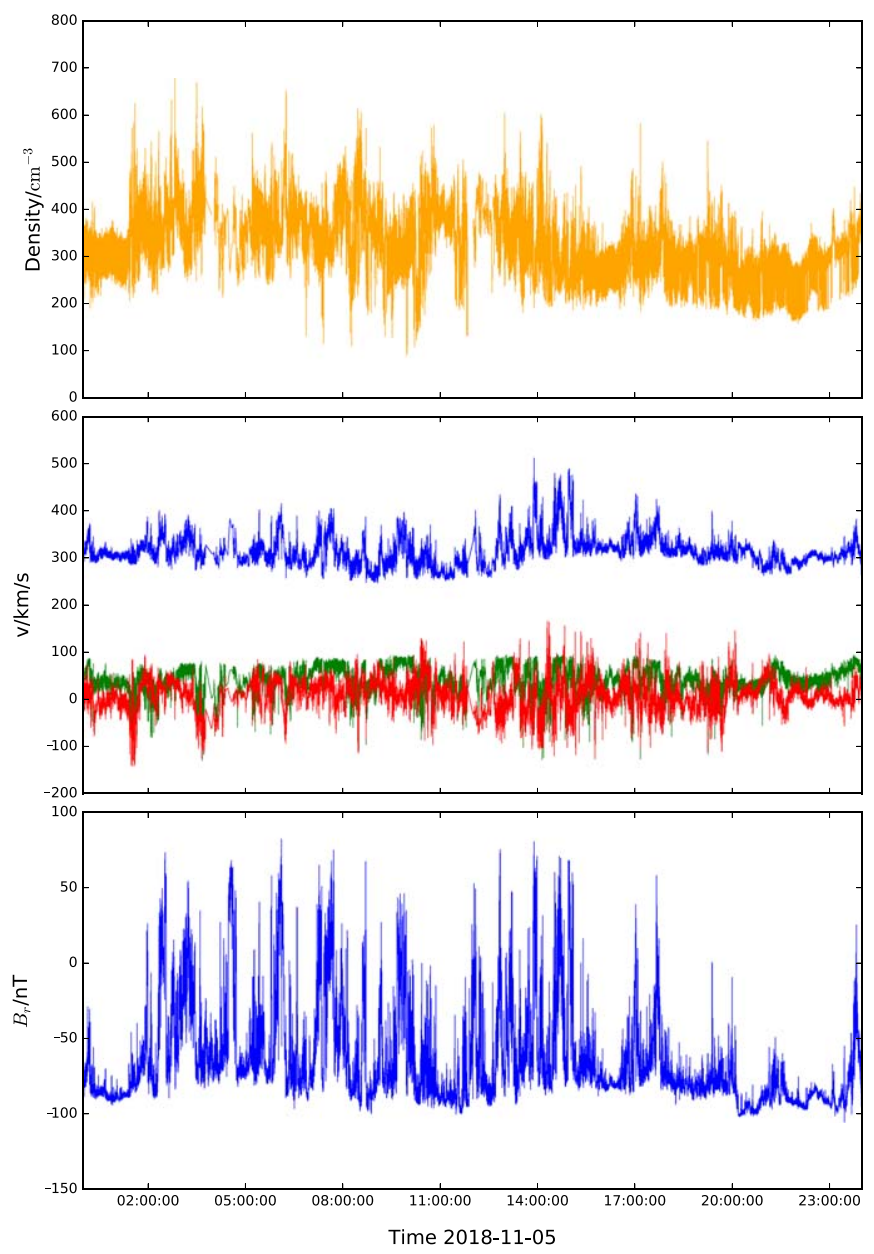

Figure 1. Time series of the encounter 1 interval. The top panel shows proton density, the middle panel shows proton velocity moments in RTN coordinates from SPC (blue, green, and red being radial, tangential, and normal, respectively), and the bottom panel shows radial component of the magnetic field.

measurement cadence and was corrected for an approximately $2.6 \mathrm{~s}$ timing offset. Large unphysical spikes were also removed, and any data gaps linearly interpolated over. Figure 1 shows particle and magnetic field data for this interval.

Throughout the analysis, we make use of wavelet transform representations of various quantities. A wavelet transform of a discrete time series, $x\left(t_{i}\right)$, is defined as (Torrence \& Compo 1998; Addison 2017)

$$
W(s, t)=\sum_{i=0}^{N-1} x\left(t_{i}\right) \psi\left(\frac{t_{i}-t}{s}\right),
$$

where $W(s, t)$ is the wavelet coefficient at scale $s$ and time $t$, $\psi(t, s)$ the wavelet function, and $\left\{t_{i}\right\}$ the set of measurement times. We use a Morlet wavelet (Farge 1992) as our wavelet function (written here unnormalized):

$$
\psi(t)=\pi^{\frac{1}{4}} e^{-\frac{1}{2} t^{2}} e^{i \sigma t},
$$

where $\sigma$ is an adjustable parameter taken here to be 6 that represents the frequency of the wavelet. We convert from dilation scale $s$ to physical (spacecraft) frequency $f$ using

$$
f=\frac{\sigma}{2 \pi \Delta t s}
$$


where $\Delta t$ is the measurement cadence. In this work, we use 24 logarithmically spaced wavelet scales $s$, from $s_{\min }=2$ to $s_{\max }=5792.62$.

First, we compute a scale and time dependent local mean magnetic field $\boldsymbol{B}_{0}(s, t)$ as

$$
\boldsymbol{B}_{0}(s, t)=\sum_{i=0}^{N-1} \boldsymbol{B}\left(t_{i}\right)\left|\psi\left(\frac{t_{i}-t}{s}\right)\right|,
$$

where the kernel $|\psi|$ is normalized to unity at each scale $s$, similar to Horbury et al. (2008) and Podesta (2009). This convolution of $\boldsymbol{B}(t)$ with $|\psi|$ can be intuitively understood as a smoothing of $\boldsymbol{B}(t)$ over a window whose size is determined by the width of the Morlet wavelet's Gaussian envelope, $|\psi|$, which in turn is set by the scale length, $s$. We then apply the wavelet transform 1 to the time series $\boldsymbol{v}(t)$ and $\boldsymbol{b}(t)$, which gives us the scale and time dependent fluctuations of $\delta \boldsymbol{v}(s, t)$ and $\delta \boldsymbol{b}$ $(s, t)$ (since the wavelet transform has no zero frequency component). In Equation (4), a local parallel field direction is defined, from which we can calculate the wavelet representations of the perpendicular fluctuations $\delta \boldsymbol{v}_{\perp}(s, t)$ and $\delta \boldsymbol{b}_{\perp}(s, t)$ and the perpendicular Elsässer variables:

$$
\delta \boldsymbol{z}_{\perp}^{ \pm}(s, t)=\delta \boldsymbol{v}_{\perp}(s, t) \pm \delta \boldsymbol{b}_{\perp}(s, t) .
$$

Here, $\delta \boldsymbol{b}_{\perp}(s, t)$ is measured in Alfvén units. To convert, we use a scale and time dependent density, $\rho(s, t)$, computed using Equation (4) applied to the density:

$$
\rho(s, t)=\sum_{i=0}^{N-1} \rho\left(t_{i}\right)\left|\psi\left(\frac{t_{i}-t}{s}\right)\right| .
$$

It is usual in the solar wind literature (Roberts et al. 1987; Bavassano et al. 1998; Bavassano \& Bruno 2006) to define $\delta z_{\perp}^{ \pm}$ in such a way that $\delta z_{\perp}^{+}$and $\delta z_{\perp}^{-}$always refer to outward and inward going waves, respectively, regardless of the direction of the background magnetic field. Since $z_{\perp}^{+}$and $z_{\perp}^{-}$wave packets travel anti-parallel/parallel to $\boldsymbol{B}_{0}$, respectively, a scheme of magnetic "rectification" is usually employed, flipping $\boldsymbol{B}_{0}$ as necessary. While this is useful when dealing with large-scale magnetic sectors of different polarity, it will be much clearer in the following discussion to leave the definition of $\delta z_{\perp}^{ \pm}$as is in Equation (5) and to define two new variables, $\delta \boldsymbol{w}_{\perp}^{ \pm}$, to represent strictly outgoing (+) and ingoing (-) waves, respectively:

$$
\delta \boldsymbol{w}_{\perp}^{ \pm}(s, t)=\left\{\begin{array}{ll}
\delta \boldsymbol{z}_{\perp}^{ \pm}(s, t) & \text { if } \operatorname{sgn}\left(\mathrm{B}_{0 r}(\mathrm{~s}, \mathrm{t})=-1\right. \\
\delta \boldsymbol{z}_{\perp}^{\mp}(s, t) & \text { if } \operatorname{sgn}\left(\mathrm{B}_{0 r}(\mathrm{~s}, \mathrm{t})=1\right.
\end{array},\right.
$$

where $B_{0 r}(s, t)$ is the radial component (in radial, tangential, normal (RTN) coordinates) of the scale dependent mean magnetic field defined in Equation (4). Physically, this is equivalent to the usual method of rectifying the field. Figure 2 illustrates these definitions for a situation where $P S P$ observes a field polarity reversal in an overall radially inward background field. For illustration, we have drawn this as an $S$-shaped bend, but a priori the exact field geometry is unknown.

To define switchback times, we first compute the time average over the entire interval of the radial component of the background magnetic field, $\left\langle B_{0 r}\left(s_{\max }, t\right)\right\rangle_{t}$, at the largest wavelet scale $s_{\max }$. We define the overall sense of the background magnetic field to be $\eta \equiv \operatorname{sgn}\left(\left\langle\mathrm{B}_{0 r}\left(\mathrm{~s}_{\max }, \mathrm{t}\right)\right\rangle_{t}\right)$. At each wavelet scale then, we can define a magnetic inversion or

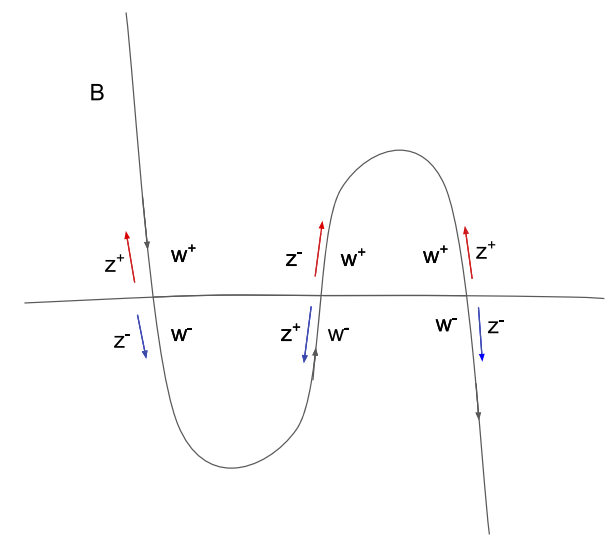

Sun

Figure 2. Schematic of a possible topology of a magnetic switchback, showing the redefinition of $\delta \boldsymbol{w}_{\perp}^{ \pm}$in terms of $\delta \boldsymbol{z}_{\perp}^{ \pm}$when $B_{r}$ changes signs.

switchback to be when $B_{0 r}(s, t)$ changes sign, relative to this largest scale background magnetic field direction. In other words, when $B_{0 r}(s, t)=-\eta$.

With these definitions in hand we can compute the normalized residual energy

$$
\begin{gathered}
\sigma_{r}(s, t)=\frac{\left|\delta \boldsymbol{v}_{\perp}(s, t)\right|^{2}-\left|\delta \boldsymbol{b}_{\perp}(s, t)\right|^{2}}{\left|\delta \boldsymbol{v}_{\perp}(s, t)\right|^{2}+\left|\delta \boldsymbol{b}_{\perp}(s, t)\right|^{2}} \\
=\frac{2 \delta \boldsymbol{z}_{\perp}^{+}(s, t) \cdot \delta \boldsymbol{z}_{\perp}^{-}(s, t)}{\left|\delta \boldsymbol{z}_{\perp}^{+}(s, t)\right|^{2}+\left|\delta \boldsymbol{z}_{\perp}^{-}(s, t)\right|^{2}},
\end{gathered}
$$

which represents the imbalance between kinetic and magnetic fluctuations or, equivalently, the alignment between the two Elsässer variables and normalized cross helicity:

$$
\begin{gathered}
\sigma_{c}(s, t)=\frac{2 \delta \boldsymbol{v}_{\perp}(s, t) \cdot \delta \boldsymbol{b}_{\perp}(s, t)}{\left|\delta \boldsymbol{v}_{\perp}(s, t)\right|^{2}+\left|\delta \boldsymbol{b}_{\perp}(s, t)\right|^{2}} \\
=\frac{\left|\delta \boldsymbol{z}_{\perp}^{+}(s, t)\right|^{2}-\left|\delta \boldsymbol{z}_{\perp}^{-}(s, t)\right|^{2}}{\left|\delta \boldsymbol{z}_{\perp}^{+}(s, t)\right|^{2}+\left|\delta \boldsymbol{z}_{\perp}^{-}(s, t)\right|^{2}},
\end{gathered}
$$

representing the alignment between velocity and magnetic field fluctuations or the imbalance between the flux of $\delta z_{\perp}^{+}$and $\delta z_{\perp}^{-}$. By analogy, we have the "rectified" cross helicity, constructed using $\delta \boldsymbol{w}_{\perp}^{ \pm}$, which we will denote $\nu_{c}$ as

$$
\nu_{c}(s, t)=\frac{\left|\delta \boldsymbol{w}_{\perp}^{+}(s, t)\right|^{2}-\left|\delta \boldsymbol{w}_{\perp}^{-}(s, t)\right|^{2}}{\left|\delta \boldsymbol{w}_{\perp}^{+}(s, t)\right|^{2}+\left|\delta \boldsymbol{w}_{\perp}^{-}(s, t)\right|^{2}}
$$

(rectification does not affect $\sigma_{r}$ ). $\nu_{c}$ is, therefore, a sensor of the ingoing versus outgoing Elsässer flux, with respect to the radial direction $\hat{\boldsymbol{r}}$, regardless of the direction of the mean magnetic field. It is helpful to think of $\sigma_{c}$ as the fractional excess of fluctuations propagating anti-parallel to $\boldsymbol{B}_{0}$ and $\nu_{c}$ as the fractional excess of fluctuations propagating away from the Sun. that

Equations (8), (10), and (12) impose the geometric constraint

$$
\begin{aligned}
& \sigma_{c}^{2}+\sigma_{r}^{2} \leqslant 1 \\
& \nu_{c}^{2}+\sigma_{r}^{2} \leqslant 1,
\end{aligned}
$$

i.e., points in $\left(\sigma_{c}\right.$ and $\left.\sigma_{r}\right)$ and $\left(\nu_{c}\right.$ and $\left.\sigma_{r}\right)$ space are constrained to lie within a circle of radius 1. For a purely Alfvénic 

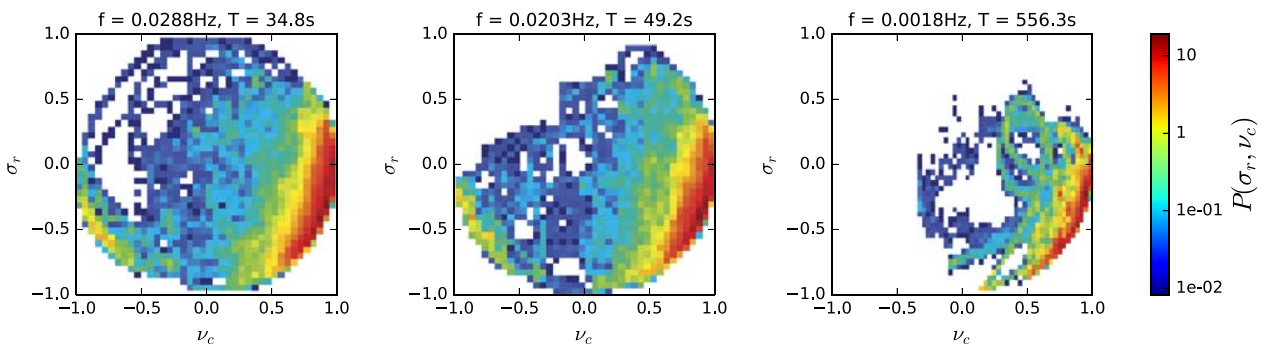

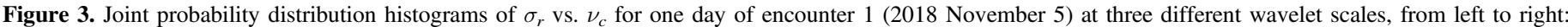
$T=35 \mathrm{~s}, T=49 \mathrm{~s}$, and $T=556 \mathrm{~s}$.
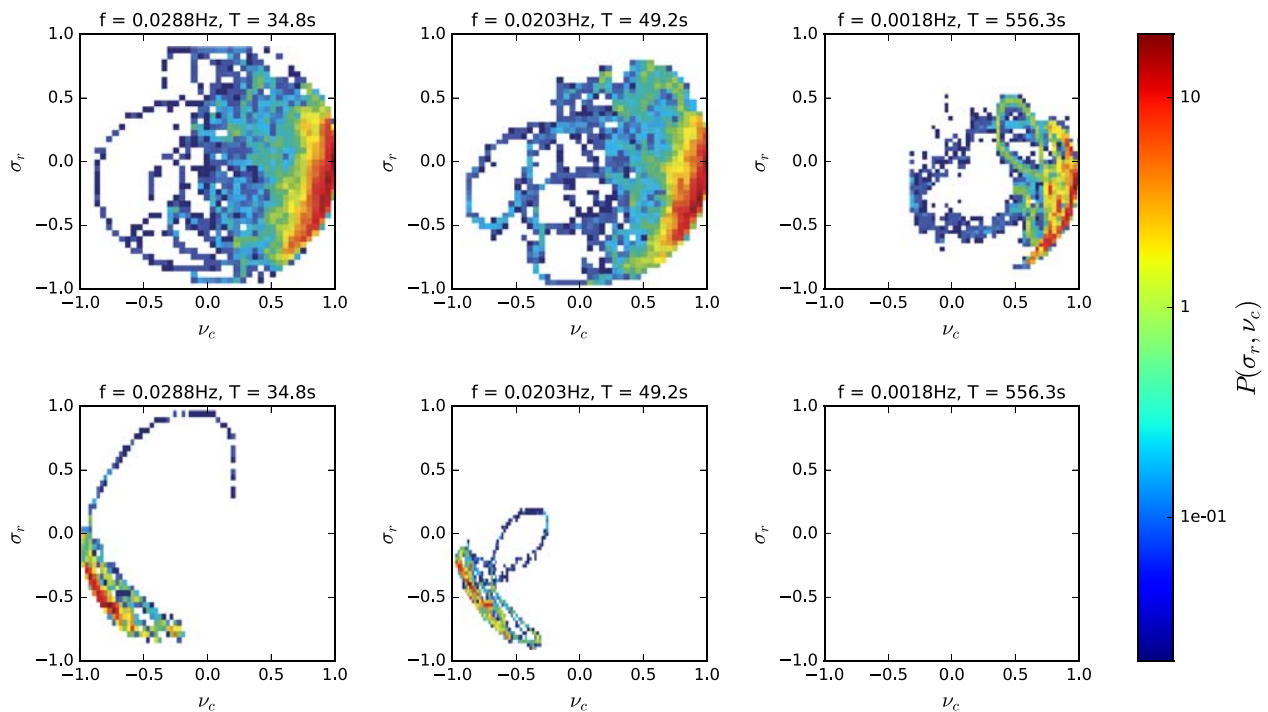

Figure 4. Joint probability distribution histograms of $\sigma_{r}$ and $\nu_{c}$ at three different wavelet scales, from left to right: $T=35 \mathrm{~s}, T=49 \mathrm{~s}$, and $T=556 \mathrm{~s}$, divided by $\theta_{B r}$ Top row: only those times on when $\theta_{B r}>160^{\circ}$, corresponding to a mainly radial field. Bottom row: only times when $\theta_{B r}<90^{\circ}$, when the radial magnetic field has locally reversed.

fluctuation, $\sigma_{r}=0$ and $\nu_{c}= \pm 1$, with + representing an outgoing wave and - an ingoing one. Values of $\left|\nu_{c}\right|<1$ represent either mixtures of ingoing and outgoing modes or mixtures of Alfvénic and non-Alfvénic fluctuations, two situations which cannot be distinguished by examining $\nu_{c}$ alone.

Finally, we define the inward and outward going Elsässer fluxes

$$
\begin{gathered}
e^{+}=\left|\delta \boldsymbol{w}_{\perp}^{+}(s, t)\right|^{2} \\
e^{-}=\left|\delta \boldsymbol{w}_{\perp}^{-}(s, t)\right|^{2} .
\end{gathered}
$$

\section{Results and Discussion}

Figure 1 shows the day-long interval during encounter 1 used in this analysis. The solar wind speed is relatively low, $v_{\mathrm{sw}} \approx 330 \mathrm{~km} \mathrm{~s}^{-1}$ (throughout encounter 1, PSP was connected mainly to the same equatorial coronal hole; Badman et al. 2020), and the radial distance is $R=0.17 \mathrm{au}$. The bottom panel shows the radial component of the magnetic field. The overall sense of the magnetic field is radially inward, but a forest of narrow, spiky switchbacks where $B_{r}$ becomes positive is clearly visible. In Figure 3, we plot joint histograms of $\sigma_{r}$ versus $\nu_{c}$ at three different wavelet scales: two short ones (35 s and $50 \mathrm{~s})$ and one relatively longer one $(560 \mathrm{~s})$. These frequencies are all well above the SPC velocity moment noise floor, which in this case corresponds to a frequency of $f \approx 0.12 \mathrm{~Hz}$ or of a scale of $T \approx 8.3 \mathrm{~s}$. All three histograms are strongly peaked in the bottom right quadrant, near the edge of the limiting circle, with maxima around $\nu_{c} \sim 0.9, \sigma_{r} \sim-0.3$, indicating highly aligned (Wicks et al. 2013) and fairly Alfvénic fluctuations. The clear signal of a "second population" at the two smaller scales is of interest and is seen as a peak in the lower left quadrant with fewer counts and similar values of $\sigma_{r} \sim-0.3$ but with negative values of $\nu_{c} \sim-0.9$. No such population is seen at the longer $560 \mathrm{~s}$ timescale (and, indeed, at any wavelet scale longer than this).

The physical origin of the negative cross helicity population can be easily understood. In Figure 4, we divide up the data according to $\theta_{B r}$, the angle between the local magnetic field, $\boldsymbol{B}_{0}(s, t)$, and the radial direction. The top row is histograms of $\sigma_{r}$ versus $\nu_{c}$ but only including times for which $\theta_{B r}>160^{\circ}-$ a mainly radial field. The second row includes only times when $\theta_{B r}<90^{\circ}$, when the magnetic field has undergone a switchback.

The negative helicity population has clearly separated and is identifiable precisely with switchback intervals. This suggests that inside switchbacks, MHD waves do indeed follow the local magnetic field; the negative cross helicity values represent what was once majority outgoing waves becoming predominantly inward propagating inside a switchback (refer again to Figure 2). This also implies that magnetic switchbacks are local kinks in the magnetic field and not due to small regions of opposite polarity at the surface of the Sun (in agreement with the conclusions in Whittlesey et al. 2019a). It is worth 

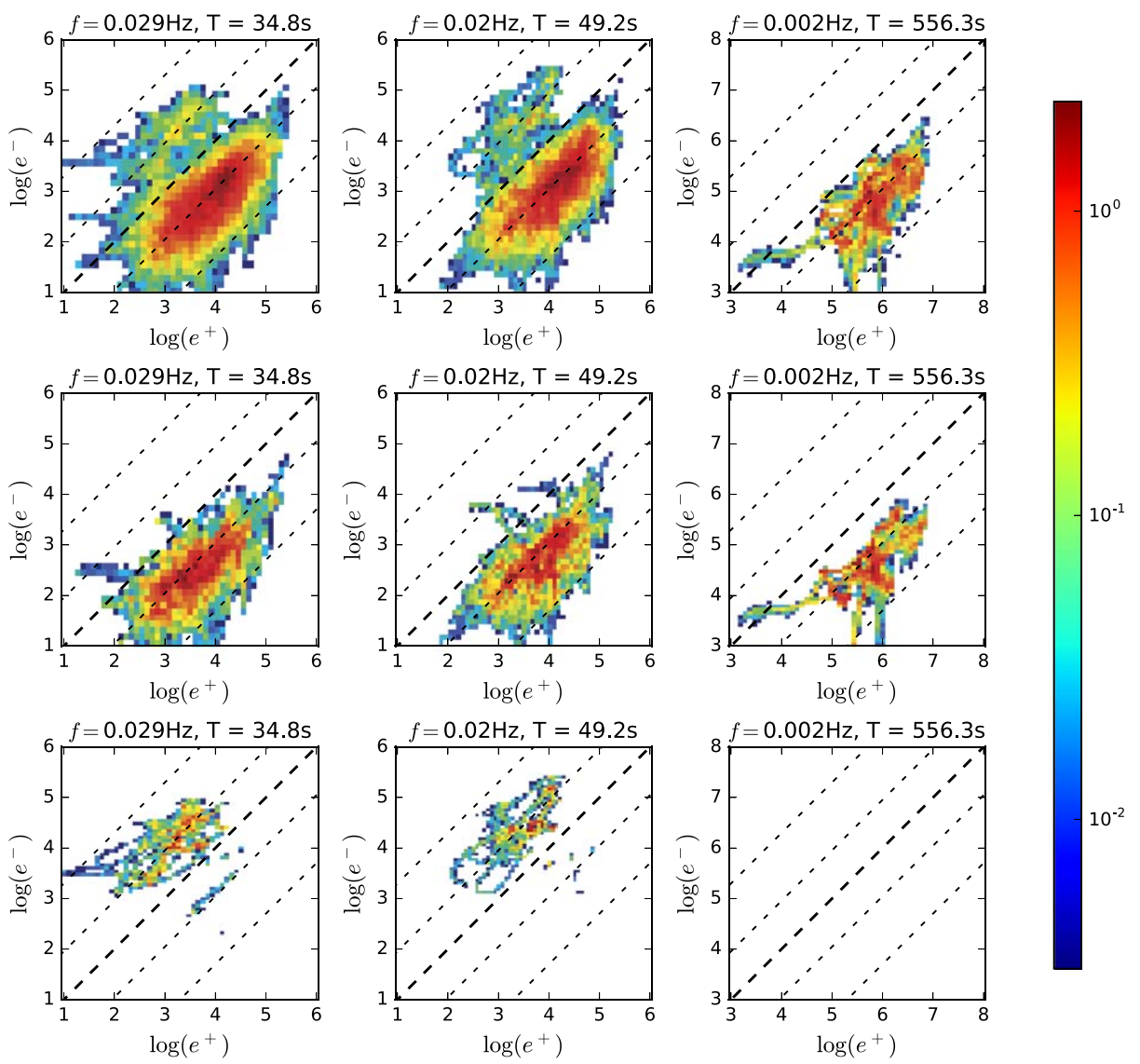

Figure 5. Joint probability distribution histograms of Elsässer power, $\log \left(e^{-}\right)$vs. $\log \left(e^{+}\right)$, at three different wavelet scales, $T=35 \mathrm{~s}, T=49 \mathrm{~s}$, and $T=556 \mathrm{~s}$; and for the same regimes as in Figure 3 (top row: all data, second row: $\theta_{B r}>160^{\circ}$, and third row: $\theta_{B r}<90^{\circ}$ ). Dashed lines represent lines of constant positive (lower right) or negative (upper left) cross helicity.

remarking here that by "inward propagating," we mean relative to the plasma frame, not the spacecraft frame, since the Alfvén velocity is much smaller than the solar wind speed.

In addition, the range of wavelet scales over which we see the negative $\nu_{c}$ population, and the scale at which it disappears, tells us something about the characteristic scale of the switchbacks at $0.17 \mathrm{au}$. In these data, switchbacks appear to last of the order of 20-100 s, and their signature has completely disappeared at scales of $\approx 300 \mathrm{~s}$ and longer (hence why the bottom right histogram in Figure 4 is empty). This is not to say switchbacks longer than this never occur. Dudok de Wit et al. (2020) present evidence that distributions of switchback deflections and residence times are power law-like, so the lack of a signature above $300 \mathrm{~s}$ in our data set is more likely a finite sampling effect rather than a hard cutoff on the timescales of switchbacks. The conclusions reached here are also in agreement with Chaston et al. (2019), who applied an MHD mode decomposition technique and found that the dominant mode both inside and outside switchbacks is always the backward propagating shear Alfvén wave, implying inward propagating plasma frame waves during switchbacks and thus kinked magnetic field lines.

Joint probability distributions of $\sigma_{r}$ and $\nu_{c}$ have been constructed many times before (Bavassano et al. 1998; Bavassano \& Bruno 2006; Bruno et al. 2007; D’Amicis et al. 2010) in a variety of solar wind conditions and heliospheric distances. In particular, Bruno et al. (2007) looked at slow wind using Helios 2 data at $0.32,0.69$, and 0.90 au. The features of their plots are broadly similar to ours (they remark there is little radial evolution in slow wind), but there is no sign of a negative cross helicity population similar to what is seen in Figure 3. This is not because switchbacks have disappeared at radial distances of $0.3 \mathrm{au}$ or greater (indeed, they have been directly observed in Helios high-speed solar wind data prior to PSP; Horbury et al. 2018) but it is a matter of scale. Given that the characteristic timescale of switchbacks at this radial distance of $0.17 \mathrm{au}$ are of the order of tens of seconds, the hour long timescale used in Bruno et al. (2007) is, certainly at smaller heliospheric distances, far too long to observe the switchbacks. It is worth noting that Bruno et al. (2007) do observe a population of negative cross helicity at larger heliospheric distances, but due to the large associated negative values of residual energy, they interpret it as being due to advected structures rather than inward propagating Alfvénic waves.

An alternative way of looking at this is shown in Figure 5, where we plot (rectified) Elsässer power, $\log \left(e^{-}\right)$versus $\log \left(e^{+}\right)$, at the same three wavelet scales and $\theta_{B r}$ regimes as in Figures 3 and 4. The diagonal dashed lines represent lines of constant cross helicity $\nu_{c}$ (from top left to bottom right, $\nu_{c}=-0.99,-0.8,0.0,0.8$, and 0.99). Again, the negative cross helicity population is seen at the two shorter scales but not the longer $560 \mathrm{~s}$ timescale. Splitting the data up by $\theta_{B r}$ isolates the negative $\nu_{c}$ population to be due to switchbacks, when $\theta_{B r}<90^{\circ}$. Both the positive and negative $\nu_{c}$ distributions are strongly peaked along lines of constant $\nu_{c}$.

Finally, in Figure 6, we show time series of $B$ and wavelet spectra of $\nu_{c}(f, t)$ and $\sigma_{c}(f, t)$ through a single switchback. The reversal in sign of $\nu_{c}$ is clearly visible, further supporting the 


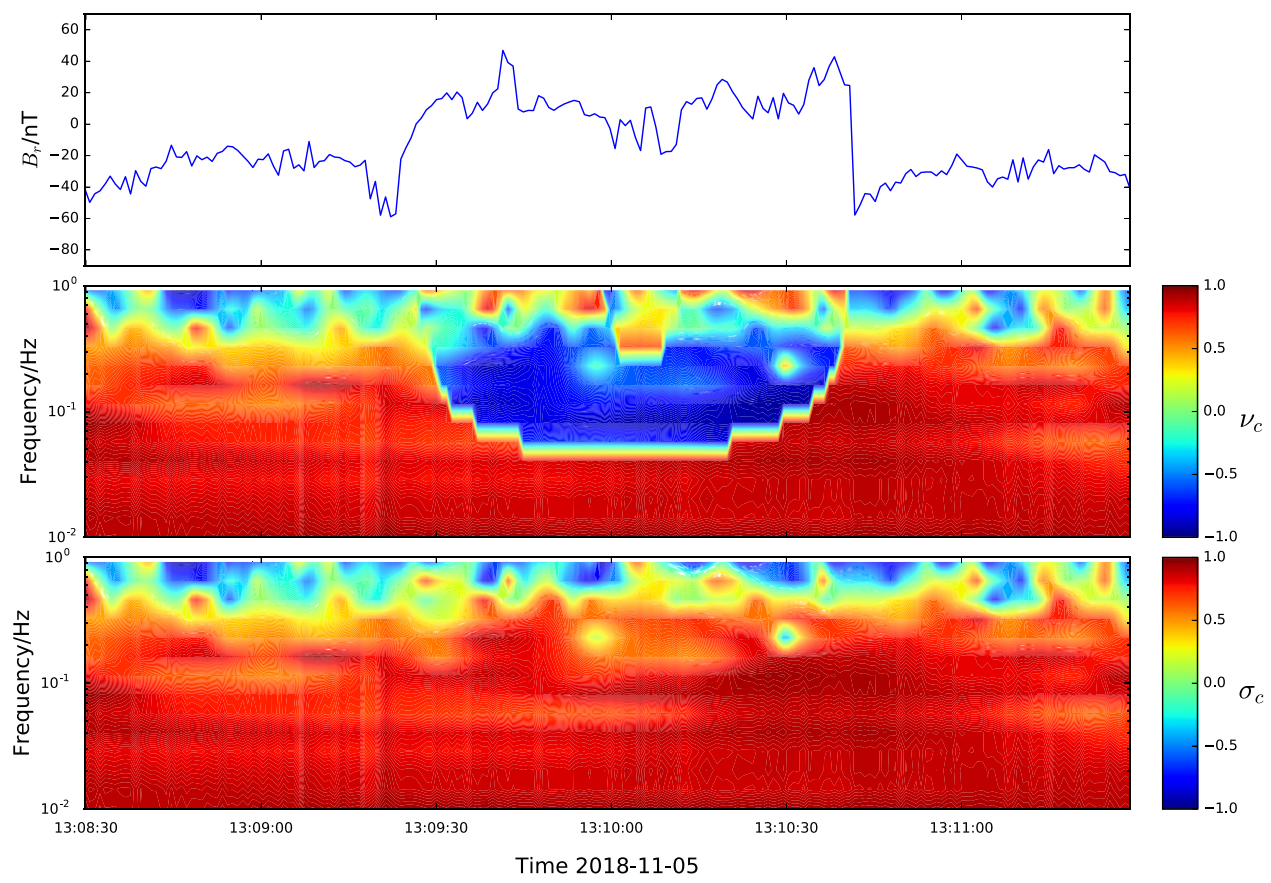

Figure 6. Behavior of cross helicity through a magnetic switchback. The top panel shows the radial magnetic field. The middle panel shows the wavelet spectrum of rectified cross helicity, $\nu_{c}(f, t)$, as a function of frequency and time. The bottom panel shows the wavelet spectrum of cross helicity, $\sigma_{c}(f, t)$.

interpretation that the MHD waves are following local field lines at their own scale through the switchback. The region of the spectrogram with negative $\nu_{c}$ does not extend to all lower frequencies (the "stepped" appearance of the feature in the $\nu_{c}$ spectrogram is a visual artifact-it is effectively the cone of influence of the edge-like feature in the magnetic field). At frequencies of $f \lesssim 4 \times 10^{-2} \mathrm{~Hz}$, the local mean field no longer sees a field reversal because it has been smoothed over a time window that is sufficiently long compared to the timescale of the switchback. Writing $\boldsymbol{B}(s, t)=\boldsymbol{B}_{0}(s, t)+\delta \boldsymbol{B}(s, t)$, one can think of the switchback as having moved from the local mean field into the fluctuations at some sufficiently large scale, and so $\nu_{c}=\sigma_{c}$ at low frequencies.

Regarding the use of $\nu_{c}$ and $\sigma_{c}$ effectively as probes of wave propagation direction, of course from Figure 6 one can come to the same physical conclusion by examining the behavior of either $\nu_{c}$ or $\sigma_{c}$. One advantage, however, of $\nu_{c}$ over $\sigma_{c}$ is that it gives us statistical information on the characteristic timescales of these events, whereas $\sigma_{c}$ does not.

\section{Conclusion}

We have considered the 2D joint distributions of normalized residual energy, $\sigma_{r}(s, t)$, and normalized rectified cross helicity, $\nu_{c}(s, t)$, during one day of PSP's first encounter as a function of the scale, $s$. The broad features of the distributions are similar to previous observations in the slow solar wind at small heliocentric distances (Bruno et al. 2007), with highly correlated and Alfvénic fluctuations $\left(\nu_{c} \sim 0.9\right.$ and $\left.\sigma_{r} \sim-0.3\right)$, but at shorter scales, a second population with $\nu_{c}<0$ is observed.

We interpret this to be due to the presence of magnetic switchbacks and confirm this by splitting the data up according to $\theta_{B r}$, the angle between the scale dependent local mean magnetic field and the radial direction, and observing the second population to only appear during switchback times. We conclude that MHD waves are following the local magnetic field inside switchbacks, even when it undergoes a large traversal. The predominantly outward propagating flux briefly becomes inward propagating during the field reversal. This also implies that these are local kinks in the magnetic field and are not due to regions of opposite polarity at the Sun's surface. Our analysis provides a useful way to distinguish between these scenarios using only in situ data. $\sigma_{c}$, as a measure of correlation between $\delta \boldsymbol{v}_{\perp}$ and $\delta \boldsymbol{b}_{\perp}$, is unaffected by the local mean field direction, showing that the switchbacks are just as Alfvénic as the surrounding wind and so switchbacks are, in some sense, an intrinsic part of it. The propagation direction, as encoded by $\nu_{c}$, is sensitive to the local mean field direction-that is, it follows it. This interpretation is further confirmed by directly looking at the Elsässer flux inside and outside switchbacks, and a case study following $\nu_{c}(f, t)$ as a function of time through a single switchback. Computing averaged values of rectified cross helicity without taking into account the reversal effect of switchbacks may result in an underestimation of $\nu_{c}$, an effect which may become more important in later PSP orbits, depending on how the distribution of switchbacks change closer to the Sun.

Finally, a wavelet representation of rectified cross helicity, $\nu_{c}(s, t)$, is seen to be a useful tool for directly observing the inward traveling flux during a large polarity reversing switchback, as well as providing statistical information about the characteristic timescales of switchbacks, which we observe to be in the range $20-100 \mathrm{~s}$ during this interval.

The authors would like to thank and acknowledge the members of the PSP mission operations and spacecraft engineering teams at the Johns Hopkins University Applied Physics Laboratory. The FIELDS and SWEAP experiments on the Parker Solar Probe spacecraft were designed and developed under NASA contract NNN06AA01C. C.H.K.C. is supported by STFC Ernest Rutherford Fellow-ship ST/N003748/2. B.D.G.C. would like to acknowledge support from NASA grants NNX17AI18G and 80NSSC19K0829. 


\section{ORCID iDs}

Michael D. McManus (D) https://orcid.org/0000-00016077-4145

Trevor A. Bowen (1) https://orcid.org/0000-0002-4625-3332

Alfred Mallet (ib https://orcid.org/0000-0001-9202-1340

Christopher H. K. Chen (i) https://orcid.org/0000-00034529-3620

Benjamin D. G. Chandran (1D https://orcid.org/0000-00034177-3328

Stuart D. Bale (iD https://orcid.org/0000-0002-1989-3596

Thierry Dudok de Wit (10 https://orcid.org/0000-00024401-0943

J. C. Kasper (iD https://orcid.org/0000-0002-7077-930X

Michael Stevens (D) https://orcid.org/0000-0002-7728-0085

Phyllis Whittlesey (ib https://orcid.org/0000-0002-7287-5098

Roberto Livi (iD https://orcid.org/0000-0002-0396-0547

Kelly E. Korreck (D) https://orcid.org/0000-0001-6095-2490

Keith Goetz (10) https://orcid.org/0000-0003-0420-3633

Peter R. Harvey (1) https://orcid.org/0000-0002-6938-0166

Marc Pulupa (iD https://orcid.org/0000-0002-1573-7457

Robert J. MacDowall (ib https://orcid.org/0000-0003-

3112-4201

David M. Malaspina (ib https://orcid.org/0000-0003-1191-1558

Anthony W. Case (iD https://orcid.org/0000-0002-3520-4041

J. W. Bonnell (ib https://orcid.org/0000-0002-0675-7907

\section{References}

Addison, P. S. 2017, The Illustrated Wavelet Transform Handbook: Introductory Theory and Applications in Science, Engineering, Medicine and Finance (Boca Raton, FL: CRC Press)

Badman, S. T., Bale, S., Martínez Oliveros, J. C., et al. 2020, ApJS, doi:10. 3847/1538-4365/ab4da7

Bale, S., Goetz, K., Harvey, P., et al. 2016, SSRv, 204, 49
Balogh, A., Forsyth, R., Lucek, E., Horbury, T., \& Smith, E. 1999, GeoRL, 26,631

Bavassano, B., \& Bruno, R. 2006, AnGeo, 24, 3179

Bavassano, B., Pietropaolo, E., \& Bruno, R. 1998, JGRA, 103, 6521

Boldyrev, S. 2005, ApJL, 626, L37

Bowen, T. A., Mallet, A., Bonnell, J. W., \& Bale, S. D. 2018, ApJ, 865, 45

Bruno, R., \& Carbone, V. 2005, LRSP, 2, 4

Bruno, R., d'Amicis, R., Bavassano, B., Carbone, V., \& Sorriso-Valvo, L. 2007, AnGeo, 25, 1913

Chandran, B. D., \& Perez, J. C. 2019, JPIPh, 85, 905850409

Chaston, C. C., Bonnell, J. W., Bale, S. D., et al. 2019, ApJS, submitted

Chen, C., Bale, S., Salem, C., \& Maruca, B. 2013, ApJ, 770, 125

Chen, C., Mallet, A., Yousef, T., Schekochihin, A., \& Horbury, T. 2011, MNRAS, 415, 3219

D’Amicis, R., Bruno, R., Pallocchia, G., et al. 2010, ApJ, 717, 474

Dudok de Wit, T., Krasnoselskikh, V. V., Bale, S. D., et al. 2020, ApJS, doi: $10.3847 / 1538-4365 / a b 5853$

Farge, M. 1992, AnRFM, 24, 395

Fox, N., Velli, M., Bale, S., et al. 2016, SSRv, 204, 7

Horbury, T., Matteini, L., \& Stansby, D. 2018, MNRAS, 478, 1980

Horbury, T. S., Forman, M., \& Oughton, S. 2008, PhRvL, 101, 175005

Karpen, J., DeVore, C., Antiochos, S., \& Pariat, E. 2017, ApJ, 834, 62

Kasper, J. C., Abiad, R., Austin, G., et al. 2016, SSRv, 204, 131

Livi, R., Larson, D. E., Kasper, J. C., et al. 2019, ApJS, submitted

Mallet, A., \& Schekochihin, A. A. 2016, MNRAS, 466, 3918

Neugebauer, M., \& Goldstein, B. E. 2013, in AIP Conf. Proc. 1539, SOLAR WIND 13, ed. G. P. Zank et al. (Melville, NY: AIP), 46

Perez, J. C., \& Chandran, B. D. 2013, ApJ, 776, 124

Podesta, J. 2009, ApJ, 698, 986

Roberts, D., Klein, L., Goldstein, M., \& Matthaeus, W. 1987, JGRA, 92, 11021

Torrence, C., \& Compo, G. 1998, BAMS, 79, 61

Uritsky, V. M., Roberts, M. A., DeVore, C. R., \& Karpen, J. T. 2017, ApJ, 837,123

Velli, M., Grappin, R., \& Mangeney, A. 1989, PhRvL, 63, 1807

Whittlesey, P., Larson, D., Kasper, J. C., et al. 2019a, ApJS, submitted

Whittlesey, P., Larson, D. E., Jalekas, J., et al. 2019b, ApJS, submitted

Wicks, R. T., Roberts, D. A., Mallet, A., et al. 2013, ApJ, 778, 177

Yamauchi, Y., Suess, S. T., Steinberg, J. T., \& Sakurai, T. 2004, JGRA, 109 A03104

Zhao, L.-L., Zank, G., Adhikari, L., et al. 2020, ApJS, doi:10.3847/1538$4365 / \mathrm{ab} 4 \mathrm{ff} 1$ 